УДК 512.54

\title{
Characterizations of Simple Linear Groups in the Class of Periodic Groups
}

\author{
Daria V. Lytkina* \\ Siberian State University of Telecommunications and Informtaion Sciencies \\ Kirova, 86, Novosibirsk, 630102 \\ Novosibirsk State University \\ Pirogova, 2, Novosibirsk, 630090 \\ Russia \\ Victor D. Mazurov ${ }^{\dagger}$ \\ Sobolev Institute of Mathematics SB RAS \\ Akad. Koptyug av., 4, Novosibirsk, 630090
}

Russia

Received 26.10.2016, received in revised form 20.12.2016, accepted 20.03.2017

This paper is a survey of some results about the structure of periodic groups saturated with finite simple groups of Lie type. It is based on a talk of authors given on the XI workshop on Group theory dedicated to the 70-th anniversary of A.Yu. Olshanskiy (Krasnoyarsk, July 27-August 2, 2016).

Keywords: linear group, periodic group, locally finite group.

DOI: 10.17516/1997-1397-2017-10-3-287-292.

\section{Introduction}

Let $G$ be a group. A family $\mathfrak{L}$ of subgroups of $G$ is said to be a local system (or a local covering) of $G$, if for any element of $G$ there is a member of $\mathfrak{L}$ containing it, and every two members of $\mathfrak{L}$ are contained in a member of $\mathfrak{L}$.

Properties of a local system of a group may be crucial to the structure of the group. For example, a group is locally finite, i. e. all of its finitely generated subgroups are finite, if and only if it has a local system consisting of finite subgroups.

As an illustration of a role of local systems in a group study, we will recall the history of classification of simple periodic groups which have a faithful matrix representation over some field (in other words, linear groups).

\section{Simple periodic linear groups}

We start with a basic theorem by Jordan stating that the order of a finite simple linear group over a field of characteristic 0 is bounded.

Theorem 1.1. [1, 36.13] There exists an integral function $J(n)$, such that, for every field $k$ of characteristic 0 and every finite simple group $G \leqslant G L(n, k)$

\footnotetext{
*daria.lytkin@gmail.com

${ }^{\dagger}$ mazurov@math.nsc.ru

(C) Siberian Federal University. All rights reserved
}

$$
|G| \leqslant J(n) .
$$


It is clear that this theorem is not true for groups over field of positive characteristics.

For example, the order of a simple finite group $L_{2}\left(2^{m}\right)=S L_{2}\left(2^{m}\right)$, that has a representation of dimension 2 over a field of order $2^{m}$, can become arbitrarily large with the growth of $m$.

Nevertheless, the class of periodic simple linear groups is quite narrow. Every of such groups is locally finite due to the following theorem.

Theorem 1.2. (I. Schur, $[1,36.14])$ A periodic linear group is locally finite.

Huge contribution to the study of locally finite groups was made by Otto Kegel, who showed that a countable locally finite simple linear group is the union of an ascending chain of finite simple groups, which means that it has a local system consisting of finite simple groups.

Theorem 1.3 (O. Kegel, [2]). A countable locally finite simple linear group is the union of an ascending sequence of finite simple subgroups.

In fact, it is easy to understand that the countability condition is unneccessary here: in fact, every finite simple periodic group is countable.

Theorem 1.4 (D. J. Winter, [3]). A periodic linear group has a unipotent normal subgroup of countable index.

In particular, every simple periodic linear group is countable.

Corollary 1.5. A periodic simple linear group is the union of an ascending sequence of finite simple subgroups.

Therefore, by Corollary 1.5 in view of the classification of finite simple groups, every simple periodic group is the union of an ascending sequence of finite simple subgroups. And every of this finite groups is isomorphic either to an alternating group of some degree, or to one of sporadic groups, or to a simple group of Lie type.

Since every alternating group of degree $n$ does not have a faithful representation of dimension smaller than $n-2$, then this sequence contains only finitely many alternating groups, and also finitely many sporadic groups, which may be discarded.

Now we are left with groups of Lie type. It is fairly obvious that their Lie ranks are bounded. Hence, modulo the classification of finite simple groups we have the following result.

Corollary 1.6 (mod CFSG). An infinite periodic simple linear group is the union of an ascending sequence of finite simple groups of Lie type whose ranks are bounded.

As it was shown by M.J. Larsen and R. Pink in 1998 году, the "mod CFSG" condition is redundant.

Theorem 1.7 (M. J. Larsen, R. Pink, [4]). There exists an integral function $J_{1}(n)$ such that, for any finite simple group $G$ posessing a faithful linear representation of dimension at most $n$ over a field $k$ we have either

1) $|G| \leqslant J_{1}(n)$, or

2) $p=\operatorname{char}(k)$ is positive and $G$ is a group of Lie type in characteristic $p$.

The paper [4] containing this theorem was properly published in 2011 (before that it was known as a preprint). It is selfsufficient and uses only basic results of algebraic geometry and representation theory of algebraic groups, but not the classification of finite simple groups.

The final chord was the following result which was obtained independently and almost simultaniously by V. V. Belyaev, A. V. Borovik, B. Hartley and G. Shute, and S. Thomas. 
Theorem 1.8 ( [5-8]). The union of an ascending sequence of finite simple groups of Lie type of bounded Lie ranks is isomorphic to a simple group of Lie type over a locally finite field.

This immediately implies that an infinite simple periodic linear group is isomorphic to a group of Lie type over a locally finite field. Since every group of Lie type is linear, we obtain the following

Corollary 1.9. We have

$\{$ Infinite simple periodic linear groups $\}=$

$$
=\{\text { Simple groups of Lie type over infinite locally finite fields }\} \text {. }
$$

This implies, that, without going into detail, every infinite simple periodic group may be written in the following way.

Corollary 1.10. We have

$\{$ Infinite simple periodic linear groups $\}=$

$$
\begin{aligned}
& =\left\{{ }^{*} X_{n}(Q) \mid X \in\{A, B, C, D, E, F, G\}, n \in \mathbb{N},\right. \\
& \quad Q \text { is an infinite locally finite field, } \\
& \quad * \text { is an empty sign, or one of the numbers } 2,3\} .
\end{aligned}
$$

Here $X_{n}$ denotes a simple Lie algebra over the complex field with which the given group is associated, that is, one of the algebras $X_{n} \in\left\{A_{n}, n \geqslant 1 ; B_{n}, n \geqslant 2 ; C_{n}, n \geqslant 3 ; D_{n}, n \geqslant\right.$ $\left.4 ; E_{6}, E_{7}, E_{8} ; F_{4} ; G_{2}\right\}$, * denotes a twist automorphism of respective order (if it exists).

In particular, any infinite simple periodic linear group posesses a local system consisting of finite simple groups of Lie type whose ranks are bounded. It is locally finite and countable.

Since every finite subgroup of a group ${ }^{*} X_{n}(Q)$ lies in a subgroup isomorphic to ${ }^{*} X_{n}(F)$, where $F$ is a finite subfield of $Q$, every group ${ }^{*} X_{n}(Q)$ is saturated with groups from the set

$$
\mathfrak{M}=\left\{{ }^{*} X_{n}(F) \mid F \text { is a finite subfield of the field } Q\right\},
$$

in the sense of the following definition.

\section{Groups saturated with given groups}

Let $\mathfrak{M}$ be a set of groups. We say that a group $G$ is saturated with groups from $\mathfrak{M}$, if any finite subgroup of $G$ is contained in a subgroup isomorphic to some member of $\mathfrak{M}$. For example, a group with local system $\mathfrak{L}$ is saturated with groups from $\mathfrak{L}$, but if $G$ is saturated with groups from a given set $\mathfrak{M}$, the set of all subgroups of $G$ isomorphic to members of $\mathfrak{M}$ do not have to be a local system for $G$.

For example, any free periodic group $B(2, n)$ of prime period $n \geqslant 665$ is saturated with groups from the set $\mathfrak{M}$, which consists of a single cyclic group of order $n$, but $B(2, n)$ is not locally finite due to Novikov and Adian.

About twenty years ago A. K. Shlöpkin posed the following conjecture which later was written to "Kourovka notebook".

Conjecture 2.1. [9, Question 14.101] A periodic group saturated with finite simple groups of Lie type of bounded Lie ranks is isomorphic to a group of Lie type over a locally finite field. 
This conjecture is valid for locally finite groups.

Proposition 2.2. If $G$ is a locally finite group which is saturated with groups from some set $\mathfrak{M}$ of finite simple groups of Lie types whose Lie ranks are bounded as a whole, then $G$ is isomorphic to a simple group of Lie type over a locally finite field.

Proof. Without loss of generality, one can assume that $G$ is infinite. Since any simple group of Lie type whose Lie rank does not exceed a given number $n$ has a faithful linear representation of degree $f(n)$ over a field, where $f$ is an integral function (see, for example, [10]), then every finitely generated subgroup of $G$ posesses a faithful linear representation over a field of degree $f(n)$. By Malcev Theorem [11, Theorem IV], $G$ itself posesses a faithful linear representation over a field.

Notice that $G$ is simple. Indeed, if $1 \neq N \unlhd G$ and $N \neq G$, then choose $x \in G \backslash N, 1 \neq y \in N$. By assumption, $\langle x, y\rangle$ is a finite subgroup which is contained in a finite simple subgroup $H \leqslant G$ and $y \in N \cap H \unlhd H$, that is $H \leqslant N$. But $x \in G \backslash N$, that is $x \notin H$, a contradiction.

Now, proposition is true by Theorem 1.8.

This proposition shows that if the Conjecture 2.1 is not true, then a counterexample should be extremely complicated, because it cannot be locally finite. In this connection, it is interesting to mention a result by A.Yu. Olshanski [12] which states that every infinite countable periodic group can be embedded in a simple periodic group with two generators (which clearly cannot be localy finite).

On the other hand, the means of studying of periodic groups which are not locally finite are fairy poor, and almost entirely rest on the fact that a periodic group generated by two involutions is finite.

We feel that more approachable is the following

Question 2.3. Let $Q$ be a locally finite field, and ${ }^{*} X_{n}(Q)$ be a group of Lie type ${ }^{*} X$ of rank $n$ over $Q$. Suppose that

$$
\mathfrak{M}=\left\{{ }^{*} X_{n}\left(Q_{\alpha}\right) \mid Q_{\alpha} \text { is a finite subfield of } Q\right\} .
$$

Is it true that any periodic group $G$ saturated with groups from $\mathfrak{M}$ is isomorphic to ${ }^{*} X_{n}\left(Q_{0}\right)$, where $Q_{0}$ is a subfield of $Q$ ?

At present time a positive answer to this question is received (in a more general context) only for groups of extremely small ranks, mainly for groups of Lie ranks 1 and 2 .

For groups of Lie rank 1

\begin{tabular}{|l|l|}
\hline$A_{1}=L_{2}$ & Rubashkin, Filippov [13] \\
\hline${ }^{2} A_{2}=U_{3}$ & Lytkina, Al. Shlöpkin [14] \\
\hline${ }^{2} B_{2}=S z$ & Filippov [15, 16] \\
\hline${ }^{2} G_{2}=$ Ree & An. Shlöpkin [17] \\
\hline
\end{tabular}

For groups of Lie rank 2

\begin{tabular}{|l|l|}
\hline$A_{2}=L_{3}$ & Lytkina, Al. Shlöpkin [14] \\
\hline$B_{2}=S_{4}=O_{5}$ & Lytkina, Mazurov; in progress \\
\hline
\end{tabular}

Groups ${ }^{2} A_{3}=U_{4} ;{ }^{2} A_{4}=U_{5} ; G_{2},{ }^{2} F_{4} ;{ }^{3} D_{4}$ and also all groups of Lie rank at least 3 are queueing for being investigated.

Perspective is fairy gloomy. But lately we have got some results which can be interpreted as bright strokes in this dark picture. 


\section{Groups of Lie type of arbitrary ranks}

Theorem 3.1 ( [18]). Let $G$ be a periodic group saturated with finite simple groups of Lie type of odd characteristics whose ranks are bounded.

Then any 2-subgroup of $G$ is locally finite.

Theorem 3.2 (Lytkina, Mazurov, in progress). Let $n \geqslant 3$ be a natural number, and $G$ a periodic group. Suppose that any finite subgroup of $G$ is contained in a subgroup of $G$ isomorphic to $B_{n}(q)=O_{2 n+1}(q)$ for some odd number $q$.

Then $G$ is isomorphic to $O_{2 n+1}(Q)$ for some locally finite field $Q$.

In particular, $G$ is locally finite.

The work was supported by the Russian Foundation for Basic Research, project 17-51-560005 Iran_a.

\section{References}

[1] C.W.Curtis, I.Reiner, Representation Theory of Finite groups and Associative Algebras, New York, London, John Wiley \& Sons, 1962.

[2] O.Kegel, Über einfache, local endliche Gruppen, Math. Z., 95(1967), 169-195.

[3] D.J.Winter, Representations of locally finite groups, Bull. Amer. Math. Soc., 74(1968), $145-148$.

[4] M.J.Larsen, R.Pink, Finite subgroups of algebraic groups, J. Amer. Math. Soc., 24(2011), no. 4, 1105-1158.

[5] V.V.Belyaev, Locally finite Chevalley groups, Studies in Group Theory, Ural'sky Nauchny Tsentr AS USSR, Sverdlovsk, 1984, 39-50 (in Russian).

[6] A.V.Borovik, Embeddings of finite Chevalley groups and periodic linear groups, Siberian Math. J., 24(1983), no. 6, 843-851.

[7] B.Hartley, G.Shute, Monomorphisms and direct limits of finite groups of Lie type, The Quaterly Journal of Mathematics Oxford, Ser. 2, 35(1984), no. 137, 49-71.

[8] S.Thomas, The classification of the simple periodic linear groups, Arch. Math., 41(1983), 103-116.

[9] Unsolved Problems in Group Theory, The Kourovka Notebook, Institut matematiki, Novosibirsk, (2014), no. 18 (in Russian).

[10] R.W.Carter, Simple groups of Lie type, London, John Wiley \& Sons, 1972.

[11] A.I.Malcev, On isomorphic matrix representations of infinite groups, Rec. Math (Mat. Sbornik), N. S., 8(50)(1940), no. 3, 405-422 (in Russian).

[12] A.Yu.Olshanskii, Embedding of countable periodic groups in simple 2-generator periodic groups, Ukrain. Mat. Zh., 43(1991), no. 7-8, 980-986.

[13] A.G.Rubashkin, K.A.Filippov, Periodic groups saturated with the groups $L_{2}\left(p^{n}\right)$, Siberian Math. J., 46(2005), no. 6, 1119-1122. 
[14] D.V.Lytkina, A.A.Shlöpkin, Periodic groups saturated with finite simple groups of the types $U_{3}$ and $L_{3}$, Algebra and Logic, 2016 (to appear).

[15] K.A.Filippov, Groups saturated with finite nonabelian groups and their extension, $\mathrm{PhD}$ thesis, Krasnoyarsk, 2005.

[16] K.A.Filippov, On periodic groups saturated by finite simple groups, Siberian Math. J., 53(2012), no. 2, 345-351.

[17] A.K.Shlöpkin, On some periodic groups saturated by finite simple groups, Mat. Tr., 1(1998), no. 1, 129-138 (in Russian).

[18] B.D.Li, D.V.Lytkina, On Sylow 2-subgroups of periodic groups saturated with finite simple groups, Sib. Math. J., (2016) (to appear).

\section{Характеризации простых линейных групп в классе периодических групп}

Дарья В. Лыткина

Сибирский государственный университет телекоммуникаций и информатики

Кирова, 86, Новосибирск, 630102

Новосибирский государственный университет

Пирогова, 2, Новосибирск, 630090

Россия

Виктор Д. Мазуров

Институт математики им. С.Л. Соболева СО РАН

пр. ак. Коптюга, 4, Новосибирск, 630090

Россия

В статъе приводится обзор результатов, связанных со структурой периодических групп, насыщенных конечными простыми группами лиева типа. Работа основана на докладе, представленном на ХІ школе-конференции по теории групп, посвящённой 70-летию А.Ю. Ольшанского (Красноярск, 27 июля - 2 августа 2016 г.).

Ключевые слова: линейная группа, периодическая группа, локально конечная группа. 